\title{
History of infertility and risk of type 2 diabetes mellitus: a prospective cohort study
}

\author{
Deirdre K. Tobias • Audrey J. Gaskins • Stacey A. Missmer • \\ Frank B. Hu • JoAnn E. Manson • Germaine M. Buck Louis • \\ Cuilin Zhang • Jorge E. Chavarro
}

Received: 20 October 2014 / Accepted: 27 December 2014 /Published online: 18 January 2015

(C) Springer-Verlag Berlin Heidelberg 2015

\begin{abstract}
Aims/hypothesis We sought to evaluate the relationship between delayed conception and type 2 diabetes risk, given that there are plausible underlying mechanisms linking the two, including inflammation and insulin resistance.

Methods Participants of the Nurses' Health Study II prospective cohort were included if they were free of chronic disease (cardiovascular disease, type 2 diabetes, cancer) at baseline. Biennial questionnaires updated information on infertility status ( $>12$ months attempted pregnancy), lifestyle characteristics
\end{abstract}

\section{K. Tobias $(\bowtie) \cdot J$. E. Manson}

Division of Preventive Medicine, Department of Medicine, Brigham and Women's Hospital and Harvard Medical School, 900 Commonwealth Avenue, Boston, MA 02215, USA

e-mail: dtobias@partners.org

D. K. Tobias • A. J. Gaskins • F. B. Hu • J. E. Chavarro Department of Nutrition, Harvard School of Public Health, Boston, MA, USA

A. J. Gaskins · S. A. Missmer · F. B. Hu • J. E. Manson •

J. E. Chavarro

Department of Epidemiology, Harvard School of Public Health,

Boston, MA, USA

S. A. Missmer · F. B. Hu • J. E. Chavarro

Channing Division of Network Medicine, Department of Medicine, Brigham \& Women's Hospital \& Harvard Medical School,

Boston, MA, USA

\section{S. A. Missmer}

Department of Obstetrics, Gynecology and Reproductive Biology, Brigham and Women's Hospital and Harvard Medical School,

Boston, MA, USA

G. M. Buck Louis $\cdot$ C. Zhang

Division of Intramural Population Health Research, Eunice Kennedy Shriver National Institute of Child Health and Human Development, Bethesda, MD, USA and several health-related outcomes. Self-reported cases of diabetes were confirmed using a follow-up questionnaire. Multivariable Cox proportional hazards models were used to compute the HRs and $95 \%$ CIs.

Results Incident type 2 diabetes occurred in 5,993 of the 112,106 participants over 24 years of follow-up (19892013). A history of infertility was reported in 27,774 (24.8\%) women and was associated with a $20 \%$ greater risk of developing diabetes, compared with those never reporting infertility (HR 1.20 [95\% CI 1.14, 1.28]), after adjusting for age, lifestyle factors, marital status, oral contraceptive use, family history of diabetes and BMI. Compared with women without a history of infertility, the causes of infertility associated with a higher diabetes risk were ovulation disorders (HR $1.43[95 \%$ CI $1.29,1.58])$ and tubal factor (HR 1.34 [95\% CI $1.13,1.58]$ ). Cervical factor (HR 1.06 [95\% CI 0.81, 1.40]) and endometriosis (HR 1.06 [95\% CI 0.89, 1.27]) were not associated, while male factor infertility was associated with a modestly higher diabetes risk (HR 1.15 [95\% CI 1.00, 1.33]). Conclusions/interpretation These novel findings suggest a history of infertility, particularly that related to ovulation disorders and tubal blockage, is significantly associated with a higher risk of type 2 diabetes.

Keywords Epidemiology · Fertility · Gestational diabetes . Infertility · Life course epidemiology · Ovulatory disorders · Prospective cohort · Reproductive health · Type 2 diabetes

$\begin{array}{ll}\text { Abbreviations } \\ \text { AHEI-2010 } & \text { Alternate Healthy Eating Index } 2010 \\ \text { GDM } & \text { Gestational diabetes mellitus } \\ \text { METs } & \text { Metabolic equivalent tasks } \\ \text { NHS II } & \text { Nurses' Health Study II } \\ \text { PCOS } & \text { Polycystic ovary syndrome }\end{array}$




\section{Introduction}

Infertility, commonly defined as failure to achieve pregnancy after more than 12 months of attempting to conceive, affects approximately $12-30 \%$ of couples, based on estimates from prospective preconception cohorts [1-4]. Obesity-related metabolic disturbances such as insulin resistance, inflammation and dyslipidaemia have been implicated in some, but not all, infertility-related conditions, in particular ovulatory disorders and polycystic ovary syndrome (PCOS) $[5,6]$. Given the common pathologies underlying many causes of infertility and type 2 diabetes, it is plausible that a history of infertility is related to a risk of diabetes many years later.

Accumulating evidence suggests a greater type 2 diabetes risk in women with PCOS $[7,8]$; however, the relationship of other causes of an infertility diagnosis, such as tubal blockages and cervical factors, with a subsequent diabetes risk is relatively unexplored. Further, few studies have addressed this question prospectively and include careful control for diabetes and possible infertility-related risk factors, such as body weight and lifestyle. Therefore, our aim was to examine the associations of a self-reported history of infertility and its attributed causes with type 2 diabetes risk in the Nurses' Health Study II (NHS II) longitudinal cohort of US women.

\section{Methods}

Study population The NHS II is a longitudinal cohort established in 1989 with the enrolment of 116,430 female nurses throughout the USA aged 24-44 years at baseline [9]. Questionnaires were distributed every 2 years aimed to capture a variety of lifestyle characteristics and update healthrelated outcomes. This study was approved by the institutional review board of the Partners Health Care System (Boston, MA, USA), with participants' consent implied biennially by the return of questionnaires. Follow-up for each questionnaire cycle was $>90 \%$ until the end of 2011 .

NHS II participants were included in our analysis if they were free of type 2 diabetes, cancer or cardiovascular disease (myocardial infarction or stroke) at the return of the 1989 baseline questionnaire. Women were also excluded from the analysis if they left the baseline infertility history questions blank. Participants were followed until their first report of type 2 diabetes, their last returned questionnaire, their death or 31 May 2013, whichever came first.

Exposure assessment A history of infertility was ascertained by a positive response to the statement 'tried to become pregnant for more than 1 year without success' on each biennial questionnaire. Those giving a 'yes' response were asked to indicate the attributed cause(s) of their infertility ('check all that apply') from the following options: tubal blockage, ovulatory disorder, endometriosis, cervical mucus factors, spouse, cause not investigated, cause not found, or other. We updated exposure status every 2 years until participants reached 35 years of age, and positive responses were carried forward; therefore, once a participant reported infertility (whether or not a cause was investigated) they maintained this exposure status until the end of follow-up. We excluded new reports of infertility after age 35 years to minimise exposure misclassification due to age-related declines in fertility [10]. We conducted a sensitivity analysis without this cutoff to include infertility reported at any age.

Prior validation of self-reported ovulatory disorder infertility was conducted in a random subset $(n=100)$ of the NHS II population citing this cause [11]. For this, participants received a supplemental questionnaire: 93\% (84/90) reported receiving diagnostic test results and/or relevant treatments. Medical records of 40 patients were reviewed and $95 \%$ of self-reported ovulatory disorder infertility was confirmed by a diagnostic test and/or treatment. In a previous validation study in our cohort (in all women-not just those reporting infertility), self-reported endometriosis was validated only in women whose diagnosis was laparoscopically confirmed [12]. Thus, we considered endometriosis as the potential cause of infertility only if the participant indicated that it was laparoscopically confirmed.

Outcome assessment Type 2 diabetes was ascertained by selfreporting of a physician's diagnosis every 2 years, and those responding positively were sent a supplemental questionnaire. Confirmed cases were defined according to the National Diabetes Data Group classification [13], by reporting at least one of the following: at least one classic symptom (excessive thirst, polyuria, unintentional weight loss, hunger) and a fasting plasma glucose concentration of $\geq 7.8 \mathrm{mmol} / 1$ (140 $\mathrm{mg} / \mathrm{dl})$ or random plasma glucose concentration of $\geq 11.1 \mathrm{mmol} / 1$ (200 mg/dl); no symptoms but at least a twofold elevation in plasma glucose concentration on more than one occasion (fasting $\geq 7.8 \mathrm{mmol} / 1$, random $\geq 11.1 \mathrm{mmol} / \mathrm{l}, 2 \mathrm{~h}$ OGTT of $\geq 11.1 \mathrm{mmol} / \mathrm{l}$ ); or hypoglycaemic medication use (insulin or oral hypoglycaemic agent). Criteria changed in June 1998 to adopt a new diagnostic threshold for fasting plasma glucose of $\geq 7.0 \mathrm{mmol} / 1$ (126 mg/dl) [14]. A subgroup validation study conducted in a similar cohort of US female nurses comparing our classification against medical records found a high level of accuracy (98\%) [15].

Covariable assessment The baseline questionnaire captured patient information regarding date of birth, race/ethnicity, height and weight, weight at age 18 years and a variety of lifestyle factors. Time-varying characteristics were updated every 2 years; these included body weight, smoking status, incident gestational diabetes, oral contraceptive or postmenopausal hormone use and menopausal status. We calculated the 
BMI as the ratio of weight in kilograms divided by height in metres squared [16]. Dietary variables, including usual alcohol intake (in g/day), were updated every 4 years beginning in 1991, using a validated semiquantitative food frequency questionnaire [17]. We estimated participants' overall diet quality based on their usual intake of the components of the Alternate Healthy Eating Index 2010 (AHEI-2010) [18]. Total physical activity was ascertained in 1989, 1991 and 1997 as the frequency of engaging in common recreational activities, from which metabolic equivalent tasks (METs) per week were derived [19]. Marital status and family history of diabetes were updated intermittently throughout follow-up.

Statistical analysis We analysed the association between history of infertility and risk of type 2 diabetes using age- and multivariable-adjusted Cox proportional hazards models to estimate the HRs and 95\% CIs. Women without a history of infertility served as the reference group. Multivariable models included updated age (continuous), smoking status (never, former, current light, current heavy smoker), AHEI-2010 diet quality score (in quintiles), total physical activity in METs per week (in quintiles), alcohol intake (0, 0.1-4.9, 5.0-9.9, $\geq 10.0 \mathrm{~g} /$ day), oral contraceptive use (current, former, never), postmenopausal hormone use (ever, never), menopausal status (pre-/perimenopausal, postmenopausal), family history of diabetes (yes, no), currently married (yes, no), race/ethnicity (White, Hispanic, African-American, Asian-American, other) and BMI (13 categories). Categories were included for any missing data for the covariates, when necessary $(<5 \%$ of data for most variables; $<10 \%$ for diet and postmenopausal hormone use). Since participants were allowed to record multiple underlying causes of infertility, the analyses of individual causes were mutually adjusted for all other causes cited. We did not adjust for parity as this may be a direct indicator of infertility itself.

Secondary analyses were conducted to assess heterogeneity by potential effect modifiers, including parity (nulliparous, $\geq$ one pregnancy of at least 6 months), a history of gestational diabetes in parous women (yes, no), White (yes, no), smoking status (ever, never), BMI category (normal, BMI <25.0; overweight, BMI 25.0-29.9; obese, BMI $\geq 30.0 \mathrm{~kg} / \mathrm{m}^{2}$ ) and family history of diabetes (parents have diabetes: yes, no). Additionally, we performed analyses stratified by birth characteristics of the respondent, including birthweight $(<2.5 \mathrm{~kg}, 2.5-3.1 \mathrm{~kg}$, $3.2-3.8 \mathrm{~kg}, \geq 3.9 \mathrm{~kg}$ ) and gestational age (full term, premature). The $p$ values for heterogeneity were derived from likelihood ratio tests comparing models with and without the interaction term added to the main effects multivariable model. We conducted sensitivity analyses including all infertility reported after age 35 years. Additionally, we assessed the potential for screening bias (i.e. women seeking a clinical diagnosis for infertility may be more likely to attend regular screenings and thus receive a diabetes diagnosis) by restricting the analysis to women who reported fasting glucose screenings on the biennial questionnaire. We used SAS version 9.1 (SAS Institute, Cary, NC, USA) for all statistical analyses.

\section{Results}

In all, 112,106 NHS II participants free of diabetes, cancer and cardiovascular disease at baseline were eligible for inclusion in our analysis. Women were on average 35 years of age with a mean BMI of $24.1 \mathrm{~kg} / \mathrm{m}^{2}$ at baseline. As indicated in Table 1, $27,774(24.8 \%)$ women reported a history of infertility either at baseline in 1989 or during follow-up. Women reporting a history of infertility were more likely to be nulliparous $(40 \%$ vs $27 \%$ ) and married ( $91 \%$ vs $86 \%$ ) at baseline compared with those without such a history, but were otherwise similar across a number of characteristics, including BMI, smoking status and healthy diet score. In participants with a history of infertility, 5,508 (19.8\%) did not seek a medical investigation of contributing causes and 265 reported infertility but did not respond to the question regarding investigation and reason for infertility. In those who did seek medical investigation $(n=22,001)$, ovulatory disorder $(n=8,052)$ and male factor infertility $(n=5,341)$ were the most common causes cited, and most women $(n=11,181)$ indicated only one underlying cause.

Incident type 2 diabetes occurred in 5,993 participants during 24 years of follow-up (after a mean of 14.1 years). Overall, a history of any infertility was significantly associated with a higher risk of type 2 diabetes (Table 2). In the age-adjusted model, a history of infertility was associated with a $28 \%$ greater risk of diabetes $(95 \%$ CI 1.21, 1.36). Results were similar after adjusting for a variety of lifestyle and diabetes risk factors, including alcohol, smoking status, diet quality, physical activity, marital status, oral contraceptive use, menopausal status and postmenopausal hormone use, family history of diabetes, and race/ethnicity (HR 1.25 [95\% CI 1.18, 1.33]). Further adjustment for BMI moderately attenuated the association, but it remained significant: there was a $20 \%$ greater risk of diabetes for women with a history of infertility compared with those without (95\% CI 1.14, 1.28).

In women with a history of infertility, of those who underwent a medical investigation, the individual attributed causes of infertility (Fig. 1) that remained significantly associated with a higher risk of type 2 diabetes after multivariable adjustment were ovulatory disorders (HR 1.43 [95\% CI 1.29, 1.58]), tubal factor (HR 1.34 [95\% CI 1.13, 1.58]) and male factor (HR 1.15 [95\% CI 1.00, 1.33]). Women with unexplained infertility had a modestly higher risk of type 2 diabetes compared with women without a history of infertility (HR 1.21 [95\% CI 1.06, 1.37]). Laparoscopically confirmed endometriosis (HR 1.06 [95\% CI 0.89, 1.27]), 'other' causes of 
Table 1 Baseline characteristics by history of infertility in 112,106 US women in NHS II from 1989 to 2013
Data are means (SD) or percentage, standardised to the age distribution of the study population

${ }^{a}$ Value is not age adjusted

${ }^{\mathrm{b}}$ A higher AHEI-2010 dietary pattern adherence score indicates greater adherence to a healthful dietary pattern

\begin{tabular}{|c|c|c|}
\hline \multirow[t]{2}{*}{ Variable } & \multicolumn{2}{|c|}{ History of infertility } \\
\hline & No & Yes \\
\hline$n$ & 84,332 & 27,774 \\
\hline $\operatorname{Age}^{\mathrm{a}}$ & $34.8(4.7)$ & $34.8(4.6)$ \\
\hline BMI $\left(\mathrm{kg} / \mathrm{m}^{2}\right)$ & $24.1(5.0)$ & $24.2(5.2)$ \\
\hline \multicolumn{3}{|l|}{ Body weight status (BMI $\left[\mathrm{kg} / \mathrm{m}^{2}\right]$ ) } \\
\hline Normal weight $(<25)$ & 70 & 69 \\
\hline Overweight (25-29) & 19 & 18 \\
\hline Obese $(\geq 30)$ & 11 & 12 \\
\hline BMI at age $18\left(\mathrm{~kg} / \mathrm{m}^{2}\right)$ & $21.3(3.3)$ & $21.2(3.4)$ \\
\hline Weight gain since age 18 years $(\mathrm{kg})$ & $7.5(10.6)$ & $8.1(11.0)$ \\
\hline \multicolumn{3}{|l|}{ Smoking status } \\
\hline Never smoker & 65 & 65 \\
\hline Past smoker & 21 & 21 \\
\hline Current smoker (low) & 12 & 12 \\
\hline Current smoker (high) & 1 & 1 \\
\hline AHEI-2010 healthy diet score (range $2.5-86.5)^{\mathrm{b}}$ & $48.7(11)$ & $48.7(11)$ \\
\hline Alcohol (g/day) & $3.1(6.1)$ & $3.0(6.0)$ \\
\hline Total physical activity (METs/week) & $24.8(36.8)$ & $25.0(36.7)$ \\
\hline Parity (previous pregnancies $\geq 6$ months) & $1.5(1.2)$ & $1.1(1.1)$ \\
\hline Nulliparous & 27 & 40 \\
\hline Married & 86 & 91 \\
\hline \multicolumn{3}{|l|}{ Oral contraceptive use } \\
\hline Never & 17 & 15 \\
\hline Past & 69 & 74 \\
\hline Current & 14 & 11 \\
\hline Family history of diabetes & 17 & 18 \\
\hline White & 92 & 92 \\
\hline \multicolumn{3}{|l|}{ Nurses' birthweight (kg) } \\
\hline$<2.5$ & 6 & 7 \\
\hline $2.5-3.1$ & 25 & 25 \\
\hline $3.2-3.8$ & 39 & 39 \\
\hline$\geq 3.9$ & 11 & 11 \\
\hline \multicolumn{3}{|l|}{ Nurses' gestational age } \\
\hline Full term & 78 & 79 \\
\hline Preterm & 6 & 7 \\
\hline \multicolumn{3}{|l|}{ Baseline causes of infertility $^{a}$} \\
\hline Cause not investigated & - & $5,508(19.8)$ \\
\hline Cause investigated & - & $22,001(79.2)$ \\
\hline Ovulatory disorder & - & 8,052 \\
\hline Endometriosis (laparoscopically confirmed) & - & 3,771 \\
\hline Cervical mucus disorder & - & 1,535 \\
\hline Tubal blockage & - & 3,041 \\
\hline Spouse & - & 5,341 \\
\hline Other cause & - & 4,623 \\
\hline Cause not found & - & 4,531 \\
\hline
\end{tabular}

infertility (HR 0.99 [95\% CI 0.86, 1.14]) and cervical factor (HR 1.06 [95\% CI 0.81, 1.40]) were not associated with diabetes risk. Women who did not investigate the cause of their infertility had a $14 \%$ greater risk of type 2 diabetes 
Table 2 History of infertility and risk of incident type 2 diabetes mellitus in 112,106 US women in NHS II from 1989 to 2013

\begin{tabular}{|c|c|c|c|c|c|c|c|c|c|c|}
\hline \multirow{2}{*}{$\begin{array}{l}\text { History of } \\
\text { infertility }\end{array}$} & \multirow[t]{2}{*}{ Participants $(n)$} & \multicolumn{3}{|c|}{ Age-adjusted model } & \multicolumn{3}{|c|}{ Multivariable model $1^{\mathrm{a}}$} & \multicolumn{3}{|c|}{ Multivariable model $2^{\mathrm{b}}$} \\
\hline & & HR & $95 \% \mathrm{CI}$ & $p$ value & $\mathrm{HR}$ & $95 \% \mathrm{CI}$ & $p$ value & HR & $95 \% \mathrm{CI}$ & $p$ value \\
\hline No & 4,244 & $1.00^{\mathrm{c}}$ & - & $<0.0001$ & $1.00^{\mathrm{c}}$ & - & $<0.0001$ & $1.00^{\mathrm{c}}$ & - & $<0.0001$ \\
\hline Yes & 1,749 & 1.28 & $1.21,1.36$ & & 1.25 & $1.18,1.33$ & & 1.20 & $1.14,1.28$ & \\
\hline
\end{tabular}

${ }^{\text {a }}$ Covariables include age (continuous), alcohol (four categories, g/day), smoking status (four categories), AHEI-2010 (quintiles), total physical activity (quintiles of METs/week), marital status (yes, no), oral contraceptive use (never, past, current), menopausal status (pre, post), postmenopausal hormone use (never, ever), family history of diabetes (yes, no) and race/ethnicity (white, African-American, Asian-American, Hispanic, other)

${ }^{\mathrm{b}}$ Covariables include those in model 1 , as well as BMI (14 categories)

${ }^{\mathrm{c}}$ Reference value

compared with women who did not report a history of infertility $(95 \%$ CI $1.02,1.27)$.

Stratified analyses indicated no statistically significant interactions for the relationship between overall infertility and diabetes risk by parity $\left(p_{\text {interaction }}=0.68\right)$, history of gestational diabetes $\left(p_{\text {interaction }}=0.63\right)$, white vs non-white $\left(p_{\text {interaction }}=\right.$ $0.37)$, smoking status $\left(p_{\text {interaction }}=0.59\right), \mathrm{BMI}\left(p_{\text {interaction }}=\right.$ 0.62 ), family history of diabetes $\left(p_{\text {interaction }}=0.92\right)$ or participants' gestational age ( $p_{\text {interaction }}=0.17$; Table 3$)$. We did find a significant interaction between infertility and the participants' own birthweight $\left(p_{\text {interaction }}=0.004\right)$ : infertility was associated with a higher diabetes risk only in women who weighed $\geq 3.2 \mathrm{~kg}$ at birth $(3.2-3.8 \mathrm{~kg}$, HR 1.30 [95\% CI $1.18,1.43] ; \geq 3.9 \mathrm{~kg}$, HR 1.34 [95\% CI $1.10,1.62]$ ). The association between a history of infertility and diabetes was null in women reporting a low birthweight $(<2.5 \mathrm{~kg}$, HR 1.10 [95\% CI 0.88, 1.37]; 2.5-3.1 kg, HR 1.03 [95\% CI 0.92, 1.15]). Results were similar when we allowed the incident infertility status to be updated after age 35 years (history of infertility yes vs no, HR 1.20 [95\% CI 1.14, 1.28]). Sensitivity analyses restricted to women reporting glucose screening within the past 2 years gave similar results, indicating that the relationship between infertility and diabetes is not due to increased health screening or surveillance practices in women with a history of infertility. Further adjustment for weight change since age 18 in the multivariable model did not alter findings (HR 1.20 [95\% CI 1.13, 1.27]).

\section{Discussion}

Findings from this large prospective cohort of US women indicate a positive relationship between a history of infertility and incident type 2 diabetes, which is independent of shared risk factors including body weight and multiple lifestyle factors. Overall, compared with women without infertility, a history of infertility prior to age 35 years was associated with a $20 \%$ greater risk of developing type 2 diabetes later in life. Ovulatory disorders and tubal factor were the underlying conditions associated with diabetes risk. Women with unexplained infertility also had a modest but significant greater risk compared with women without a history of infertility.

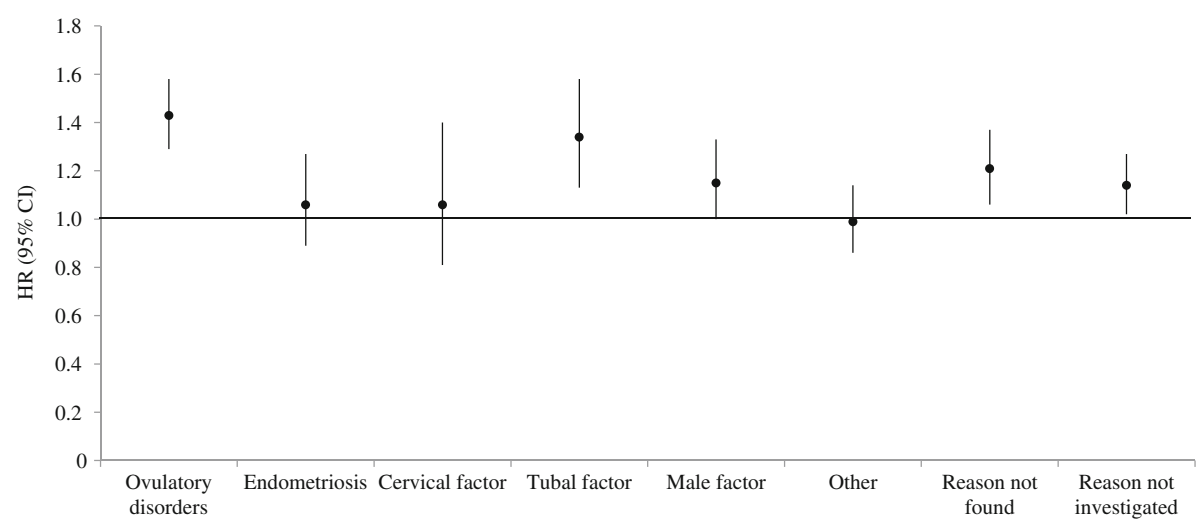

Fig. 1 Causes of infertility and risk of incident type 2 diabetes in 112,106 women in NHS II from 1989 to 2013. Estimates were obtained from multivariable models adjusted for age (continuous), alcohol (four categories, g/day), smoking status (four categories), the AHEI-2010 (quintiles), total physical activity (quintiles of METs/week), marital status (yes, no), oral contraceptive use (never, past, current), menopausal status (pre, post), postmenopausal hormone use (never, ever), family history of diabetes (yes, no), race/ethnicity (white, African-American, Asian-American, Hispanic, other) and BMI (14 categories), mutually adjusted for the presence of all other causes of infertility. Circles represent HRs and bars represent $95 \%$ CIs 
Table 3 History of infertility and risk of type 2 diabetes, stratified by diabetes risk factors
${ }^{\mathrm{a}}$ Estimated from multivariable models adjusted for age (continuous), alcohol (four categories, $\mathrm{g}$ /day), smoking status (four categories), AHEI-2010 (quintiles), total physical activity (quintiles of METs/week), marital status (yes, no), oral contraceptive use (never, past, current), menopausal status (pre, post), postmenopausal hormone use (never, ever), family history of diabetes (yes, no), race/ethnicity (White, AfricanAmerican, Asian-American, Hispanic, other) and BMI (14 categories), and mutually adjusted for the presence of all other causes of infertility

\begin{tabular}{|c|c|c|c|c|}
\hline Characteristic & Participants $(n)$ & $\mathrm{HR}^{\mathrm{a}}$ & $95 \% \mathrm{CI}^{\mathrm{a}}$ & $p_{\text {interaction }}$ value \\
\hline \multicolumn{5}{|l|}{ Parity } \\
\hline Nulliparous & 1,308 & 1.20 & $(1.04,1.39)$ & \multirow[t]{2}{*}{0.68} \\
\hline Parous & 4,685 & 1.21 & $(1.14,1.29)$ & \\
\hline \multicolumn{5}{|l|}{ History of GDM (in parous) } \\
\hline No GDM & 3,562 & 1.16 & $(1.08,1.25)$ & \multirow[t]{2}{*}{0.63} \\
\hline GDM & 839 & 1.17 & $(1.00,1.38)$ & \\
\hline \multicolumn{5}{|l|}{ Race/ethnicity } \\
\hline White & 5,362 & 1.19 & $(1.12,1.27)$ & \multirow[t]{2}{*}{0.37} \\
\hline Non-white & 631 & 1.34 & $(1.11,1.62)$ & \\
\hline \multicolumn{5}{|l|}{ Smoking status } \\
\hline Never smoker & 3,712 & 1.23 & $(1.14,1.32)$ & \multirow[t]{2}{*}{0.59} \\
\hline Ever smoker & 2,281 & 1.18 & $(1.07,1.29)$ & \\
\hline \multicolumn{5}{|l|}{ BMI $\left(\mathrm{kg} / \mathrm{m}^{2}\right)$} \\
\hline Normal weight $(<25.0)$ & 378 & 1.23 & $(0.97,1.54)$ & \multirow[t]{3}{*}{0.62} \\
\hline Overweight (25.0-29.9) & 1,054 & 1.24 & $(1.08,1.42)$ & \\
\hline Obese $(\geq 30.0)$ & 4,541 & 1.18 & $(1.11,1.26)$ & \\
\hline \multicolumn{5}{|l|}{ Family history of diabetes } \\
\hline Yes & 2,778 & 1.20 & $(1.11,1.30)$ & \multirow[t]{2}{*}{0.92} \\
\hline No & 3,205 & 1.21 & $(1.11,1.32)$ & \\
\hline \multicolumn{5}{|l|}{ Birthweight (kg) } \\
\hline$<2.5$ & 579 & 1.10 & $(0.88,1.37)$ & \multirow[t]{4}{*}{0.004} \\
\hline $2.5-3.1$ & 1,704 & 1.03 & $(0.92,1.15)$ & \\
\hline $3.2-3.8$ & 2,149 & 1.30 & $(1.18,1.43)$ & \\
\hline$\geq 3.9$ & 598 & 1.34 & $(1.10,1.62)$ & \\
\hline \multicolumn{5}{|l|}{ Gestational age } \\
\hline Full term & 4,808 & 1.21 & $(1.14,1.29)$ & \multirow[t]{2}{*}{0.17} \\
\hline Premature & 488 & 1.02 & $(0.81,1.29)$ & \\
\hline
\end{tabular}

Few studies have prospectively evaluated the relationship between overall infertility and incident type 2 diabetes in women. A previous analysis conducted in Dutch women in the European Prospective Investigation into Cancer and Nutrition ('EPIC') cohort did not observe an association between ever seeking consultation for fertility problems and type 2 diabetes risk (HR 1.05 [95\% CI 0.84, 1.32]) [20]. However, this analysis included both prevalent and incident diabetes cases, and self-reporting of fertility problems was obtained at baseline at an average age of 57 years. Associations in the literature between nulliparity (a potential marker of infertility) and diabetes risk are mixed, with some studies suggesting a greater risk in nulliparous women compared with parous women [21], and others indicating the opposite [22, 23] or a null association [24-26]. Parity, however, may be an inappropriate surrogate for infertility because it cannot account for pregnancy intention. In addition, within our study population, most infertility was reported in parous women (i.e. secondary and not primary infertility).

Our findings are consistent with a previous analysis in the NHS II cohort of infertility and risk of gestational diabetes mellitus (GDM) in parous women [27]. Here, we found that prepregnancy infertility was associated with a $39 \%$ higher risk of GDM (RR 1.39 [95\% CI 1.24, 1.57]). Similar to our findings with type 2 diabetes, both ovulatory disorders (RR 1.52 [95\% CI 1.23, 1.87]) and tubal factor (RR 1.83 [95\% CI 1.20, 2.77]) were directly associated with GDM risk. GDM is significantly associated with the development of type 2 diabetes; thus, the relationship between infertility and these diabetes outcomes may reflect similar mechanisms. However, we observed a significant relationship between a history of infertility (compared with no infertility) with type 2 diabetes risk, even in parous women without GDM, suggesting that the relationship between infertility and type 2 diabetes is not entirely mediated by GDM.

Potential explanations for our findings include a causal relationship in which the experience of infertility or its cause directly leads to an increased diabetes risk. Alternatively, the association may represent shared aetiologies between these events, indicating common risk factors or similar underlying pathologies. Furthermore, the explanation for the observed associations may differ according to the cause of infertility. Our analyses carefully controlled for lifestyle and healthrelated diabetes risk factors, but we were unable to rule out the possibility of a shared metabolic dysfunction between infertility and diabetes (i.e. 'common soil'). For example, PCOS 
is a metabolic disorder characterised by ovulatory dysfunction, insulin resistance and hyperinsulinaemia, and women with PCOS have an elevated diabetes risk $[8,28]$. The causality or direction of the relationship between irregular ovulation or anovulation and insulin resistance in PCOS is unclear, although it is believed that underlying hormonal imbalances, such as elevated endogenous androgen levels, may play a role [29]. Differences in endogenous hormone levels, particularly elevated testosterone levels, have also been implicated in type 2 diabetes in women [30]. Additionally, improved ovulatory function and menstrual cycle regularity has been observed in women with PCOS receiving oral hypoglycaemic drugs including thiazolidinediones [31], metformin [31], D-chiro-inositol [32] and acarbose [33, 34], further supporting roles for insulin resistance and glycaemic control in ovulatory function [35]. PCOS was not reliably ascertained in NHS II, so we cannot assess the role it may play in the observed association between ovulatory dysfunction and type 2 diabetes risk in our cohort. However, in support of a role for PCOS in the observed association, long and irregular menstrual cycles (a manifestation of PCOS) were previously linked to a higher risk of type 2 diabetes in this cohort [36].

With respect to infertility due to tubal disease, inflammation may be one possible biologically relevant pathway between this condition and diabetes. Tubal disease is often the result of progression of a local infection to pelvic inflammatory disease [37]. Although local inflammation begetting systemic inflammation and subsequent diabetes is plausible, and consistent with our previous finding linking tubal factor infertility with GDM, we did not identify any previous reports of a link between these conditions. Thus, further research is needed to confirm this novel association. The observed association between male factor infertility and diabetes risk was unexpected, and may be due to male partners being more likely to have been screened for fertility issues in couples experiencing delayed conception than in couples without such experience. Thus, women reporting a history of infertility would be more likely than women not reporting a history of infertility to have a male partner diagnosed with male factor infertility. For women reporting unexplained fertility, it is unknown to what extent they had been examined for possible causes of their delayed conception, and thus we are unable to determine the absence or presence of the other causes for infertility (e.g. ovulatory disorders, endometriosis).

No previous studies have evaluated the relationship between infertility and diabetes risk by participant birthweight or gestational age. Stratifying by birthweight indicated that the diabetes risk associated with a history of infertility was limited to women with birthweights $\geq 3.2 \mathrm{~kg}$. We did not observe an association between a history of infertility and diabetes in women with a lower birthweight $(<3.2 \mathrm{~kg})$. Similarly, we did not observe an association between a history of infertility and diabetes in women who were born prematurely. We speculate that this may indicate heterogeneity in the aetiologies of infertility. For example, infertility in women of low birthweight or born prematurely may be more likely to be a consequence of the low birthweight or premature birth itself (e.g. underdevelopment) or of the underlying causes of low birthweight or premature birth (such as intrauterine exposures and genetic factors) rather than of the metabolic factors associated with diabetes risk. For example, previous studies have shown that low-birthweight infant girls display markers of altered follicular function and ovarian development [38]. Additional research is therefore necessary to confirm our finding, which may have also been due to chance.

Limitations of this analysis include the self-reporting of infertility and its underlying causes. Further validation studies are warranted to determine the accuracy of self-reporting, although ovulatory infertility was previously validated against medical records in our cohort. In addition, it is unknown how much variation there was in diagnostic practices for the investigation of infertility between participants' physicians in the 1980s and 1990s, when most infertility was reported. A patient may have multiple conditions contributing to their failure to conceive but may only be aware of the condition that was identified first. For example, if a patient responds positively to empirical treatment with clomiphene for suspected anovulation and subsequently conceives, she may never become aware of undiagnosed endometriosis, unilateral tubal factor or mild male factor infertility. Information regarding change in partner status is also unavailable in this cohort. Random misclassification of fertility status would underestimate our findings; it is unknown whether specific participant characteristics are associated with the accuracy of selfreported infertility in our cohort at this time. Replication of these findings in cohorts with a consistent evaluation of infertility across participants is necessary to corroborate the underlying causes of infertility. Second, most of our cohort is white and the generalisability of our findings to other racial and ethnic groups warrants further investigation. Third, we have limited information on fertility treatments in the NHS II population and therefore cannot assess the role that these regimens may have had as intermediates. Finally, although we carefully controlled for BMI, other anthropometric indices such as waist circumference may be independently related to both lower fertility and a higher risk of diabetes; thus, residual confounding by central adiposity is possible [39, 40]. The borderline significant association between male factor infertility and diabetes risk may also reflect residual confounding, as we were unable to control for spouses' BMI, which may correlate with participants' risk factor status.

This is the first large cohort study to prospectively assess the relationship between infertility and its underlying causes with incident type 2 diabetes risk. Our repeated, prospective assessment of infertility status throughout follow-up reduces measurement error, and the long study duration allowed us to 
identify over 5,000 incident type 2 diabetes cases. The NHS II questionnaires identified several validated lifestyle and healthrelated variables, thus allowing us to control for a variety of potential confounders.

Our findings indicate a positive relationship between a history of infertility and type 2 diabetes risk in women, compared with those without such history, independent of several common risk factors. These results also suggest that the underlying causes of infertility may differ in aetiology; thus, their relation to diabetes risk should be evaluated separately. Clinically, these findings may point to a role for underlying insulin resistance and metabolic dysfunction in some causes of infertility, and could reveal potential targets for fertility treatment. Further prospective studies are necessary to corroborate these novel findings, particularly with respect to ovulation disorders and tubal disease.

Funding Financial support was provided by grants from the National Cancer Institute (UM1 CA176726) and the National Institutes of Health's National Institute of Diabetes and Digestive and Kidney Diseases (DK58845). DKT and FBH are supported by the American Diabetes Association Mentor-Based Postdoctoral Fellowship Award 7-12-MN-34. $\mathrm{CZ}$ and GMBL are supported by the Intramural Research Programme of the Eunice Kennedy Shriver National Institute of Child Health \& Human Development, National Institutes of Health.

Duality of interest The authors declare that there is no duality of interest associated with this manuscript.

Contribution statement DKT planned and performed the data analysis and wrote the manuscript. AJG reviewed the data analysis and edited the manuscript. SAM planned the data analysis and reviewed and edited the manuscript. FBH contributed to the study design and reviewed and edited the manuscript. JEM contributed to the study design and reviewed and edited the manuscript. GMBL contributed to data interpretation and reviewed and edited the manuscript. $\mathrm{CZ}$ contributed to the study design and reviewed and edited the manuscript. JEC planned the data analysis, reviewed and edited the manuscript, and is the guarantor of this work. All authors approved the final version of the manuscript.

\section{References}

1. Buck Louis GM, Sundaram R, Schisterman EF et al (2012) Heavy metals and couple fecundity, the LIFE Study. Chemosphere 87:12011207

2. Buck Louis GM, Schisterman EF, Sweeney AM et al (2011) Designing prospective cohort studies for assessing reproductive and developmental toxicity during sensitive windows of human reproduction and development - the LIFE Study. Paediatr Perinat Epidemiol 25:413-424

3. Wise LA, Rothman KJ, Mikkelsen EM, Sorensen HT, Riis A, Hatch EE (2010) An internet-based prospective study of body size and time-to-pregnancy. Hum Reprod 25:253-264

4. Hull MG, Glazener CM, Kelly NJ et al (1985) Population study of causes, treatment, and outcome of infertility. Br Med J (Clin Res Ed) 291:1693-1697
5. Lim SS, Norman RJ, Davies MJ, Moran LJ (2013) The effect of obesity on polycystic ovary syndrome: a systematic review and meta-analysis. Obes Rev 14:95-109

6. Bhathena RK (2011) Insulin resistance and the long-term consequences of polycystic ovary syndrome. J Obstet Gynaecol 31:105110

7. Caserta D, Adducchio G, Picchia S, Ralli E, Matteucci E, Moscarini M (2014) Metabolic syndrome and polycystic ovary syndrome: an intriguing overlapping. Gynecol Endocrinol 30:397-402

8. Celik C, Tasdemir N, Abali R, Bastu E, Yilmaz M (2014) Progression to impaired glucose tolerance or type 2 diabetes mellitus in polycystic ovary syndrome: a controlled follow-up study. Fertil Steril 101:11231128

9. Solomon CG, Willett WC, Carey VJ et al (1997) A prospective study of pregravid determinants of gestational diabetes mellitus. JAMA 278:1078-1083

10. Wallace WH, Kelsey TW (2010) Human ovarian reserve from conception to the menopause. PLoS One 5:e8772

11. Rich-Edwards JW, Goldman MB, Willett WC et al (1994) Adolescent body mass index and infertility caused by ovulatory disorder. Am J Obstet Gynecol 171:171-177

12. Missmer SA, Hankinson SE, Spiegelman D, Barbieri RL, Marshall LM, Hunter DJ (2004) Incidence of laparoscopically confirmed endometriosis by demographic, anthropometric, and lifestyle factors. Am J Epidemiol 160:784-796

13. National Diabetes Data Group (1979) Classification and diagnosis of diabetes mellitus and other categories of glucose intolerance. Diabetes 28:1039-1057

14. (1997) Report of the Expert Committee on the Diagnosis and Classification of Diabetes Mellitus. Diabetes Care 20:1183-1197

15. Manson JE, Rimm EB, Stampfer MJ et al (1991) Physical activity and incidence of non-insulin-dependent diabetes mellitus in women. Lancet 338:774-778

16. Rimm EB, Stampfer MJ, Colditz GA, Chute CG, Litin LB, Willett WC (1990) Validity of self-reported waist and hip circumferences in men and women. Epidemiology 1:466-473

17. Willett W (1998) Nutritional epidemiology. Oxford University Press, New York

18. Chiuve SE, Fung TT, Rimm EB et al (2012) Alternative dietary indices both strongly predict risk of chronic disease. J Nutr 142: 1009-1018

19. Wolf AM, Hunter DJ, Colditz GA et al (1994) Reproducibility and validity of a self-administered physical activity questionnaire. Int J Epidemiol 23:991-999

20. Elbers CC, Onland-Moret NC, Eijkemans MJ, Wijmenga C, Grobbee DE, van der Schouw YT (2011) Low fertility and the risk of type 2 diabetes in women. Hum Reprod 26:3472-3478

21. Hanley AJ, McKeown-Eyssen G, Harris SB et al (2002) Association of parity with risk of type 2 diabetes and related metabolic disorders. Diabetes Care 25:690-695

22. Kritz-Silverstein D, Barrett-Connor E, Wingard DL (1989) The effect of parity on the later development of non-insulin-dependent diabetes mellitus or impaired glucose tolerance. N Engl J Med 321:12141219

23. Mueller NT, Mueller NJ, Odegaard AO et al (2013) Higher parity is associated with an increased risk of type-II diabetes in Chinese women: the Singapore Chinese Health Study. BJOG 120:1483-1489

24. Liu B, Jorm L, Banks E (2010) Parity, breastfeeding, and the subsequent risk of maternal type 2 diabetes. Diabetes Care 33:1239-1241

25. Manson JE, Rimm EB, Colditz GA et al (1992) Parity and incidence of non-insulin-dependent diabetes mellitus. Am J Med 93:13-18

26. Gunderson EP, Lewis CE, Tsai AL et al (2007) A 20 year prospective study of childbearing and incidence of diabetes in young women, controlling for glycemia before conception: the Coronary Artery Risk Development in Young Adults (CARDIA) Study. Diabetes 56: 2990-2996 
27. Tobias DK, Chavarro JE, Williams MA et al (2013) History of infertility and risk of gestational diabetes mellitus: a prospective analysis of 40,773 pregnancies. Am J Epidemiol 178:1219-1225

28. Huang G, Coviello A (2012) Clinical update on screening, diagnosis and management of metabolic disorders and cardiovascular risk factors associated with polycystic ovary syndrome. Curr Opin Endocrinol Diabetes Obes 19:512-519

29. Randeva HS, Tan BK, Weickert MO et al (2012) Cardiometabolic aspects of the polycystic ovary syndrome. Endocr Rev 33: $812-841$

30. Ding EL, Song Y, Malik VS, Liu S (2006) Sex differences of endogenous sex hormones and risk of type 2 diabetes: a systematic review and meta-analysis. JAMA 295:1288-1299

31. Tang T, Lord JM, Norman RJ, Yasmin E, Balen AH (2010) Insulinsensitising drugs (metformin, rosiglitazone, pioglitazone, D-chiro-inositol) for women with polycystic ovary syndrome, oligo amenorrhoea and subfertility. Cochrane Database Syst Rev, Issue 2, Art. no.: CD003053. doi:10.1002/14651858.CD003053.pub4

32. Galazis N, Galazi M, Atiomo W (2011) D-Chiro-inositol and its significance in polycystic ovary syndrome: a systematic review. Gynecol Endocrinol 27:256-262

33. Sonmez AS, Yasar L, Savan K et al (2005) Comparison of the effects of acarbose and metformin use on ovulation rates in clomiphene citrate-resistant polycystic ovary syndrome. Hum Reprod 20:175179

34. Penna IA, Canella PR, Reis RM, Silva de Sa MF, Ferriani RA (2005) Acarbose in obese patients with polycystic ovarian syndrome: a double-blind, randomized, placebo-controlled study. Hum Reprod 20: 2396-2401

35. Nestler JE, Jakubowicz DJ, Evans WS, Pasquali R (1998) Effects of metformin on spontaneous and clomiphene-induced ovulation in the polycystic ovary syndrome. N Engl J Med 338:1876-1880

36. Solomon CG, Hu FB, Dunaif A et al (2001) Long or highly irregular menstrual cycles as a marker for risk of type 2 diabetes mellitus. JAMA 286:2421-2426

37. Kodaman PH, Arici A, Seli E (2004) Evidence-based diagnosis and management of tubal factor infertility. Curr Opin Obstet Gynecol 16: 221-229

38. Sir-Petermann T, Marquez L, Carcamo M et al (2010) Effects of birth weight on anti-mullerian hormone serum concentrations in infant girls. J Clin Endocrinol Metab 95:903-910

39. Robker RL, Wu LL, Yang X (2011) Inflammatory pathways linking obesity and ovarian dysfunction. J Reprod Immunol 88:142-148

40. Tersigni C, Di Nicuolo F, D'Ippolito S, Veglia M, Castellucci M, Di Simone N (2011) Adipokines: new emerging roles in fertility and reproduction. Obstet Gynecol Surv 66:47-63 